\title{
Unanswered Questions in Pregnancy Associated Acute Coronary Syndrome: An Argument for the Creation of Specific Guidelines for the Management of This Condition
}

\author{
Mallikarjuna MRE ${ }^{1^{*}}$ and Ganga $\mathrm{V}^{2}$ \\ ${ }^{1}$ Fellow in Cardiovascular Diseases, University of Arkansas for Medical Sciences, USA \\ ${ }^{2}$ Internal Medicine, St. Vincent Health Care System, USA
}

"Corresponding author: Mallikarjuna MRE, Fellow in Cardiovascular Diseases, University of Arkansas for Medical Sciences, USA, Tel: 501-686-7438; E-mail: mmedupuganti@gmail.com

Received date: June 19, 2016; Accepted date: June 20, 2016; Published date: June 27, 2016

Copyright: (c) 2016 Mallikarjuna MRE, et al. This is an open-access article distributed under the terms of the Creative Commons Attribution License, which permits unrestricted use, distribution, and reproduction in any medium, provided the original author and source are credited.

\section{Editorial}

Pregnancy associated acute myocardial infarction is extremely uncommon; the reported incidence is one event for every 160,000 deliveries [1]. This makes it very difficult to study the condition systematically.

In the absence of specific recommendations, the clinician often times has to extrapolate existing literature on the management of acute coronary syndrome in the general population to pregnant patients. The existing literature on the management of acute coronary syndrome deals with middle aged to elderly individuals who are predominantly male. It is therefore not difficult to understand why it is less than optimal to use this information to manage young females. Furthermore, the causes of acute myocardial infarction in the general population are vastly different from those encountered in pregnant females. While atherosclerotic coronary artery disease is the leading cause of ischemic heart disease in the general population, the causes of myocardial ischemia in pregnancy are many [2].

The most common cause of pregnancy associated acute myocardial infarction is debatable. A review of the existing literature suggests that spontaneous coronary artery dissection is the most common cause of myocardial ischemia in this population [2]. Atherosclerotic coronary artery disease is probably just as common as coronary dissection [2]. It is important to recognize other rare causes also occur, these include coronary arterial spasm and hypercoagulable conditions that lead to arterial thrombosis [2]. This is another reason why extrapolation of existing guidelines proves to be inadequate because of their focus on atherosclerotic coronary arterial disease. While plaque disruption is the most common pathophysiologic mechanism underlying acute coronary syndrome in atherosclerotic coronary arterial disease other mechanism come into play in other condition which are more common in pregnancy associated acute myocardial infarction.

In spontaneous coronary arterial dissection, the most common mechanism is the occurrence of intramural haemorrhage in the arterial wall [3]. This leads to a dissection of the arterial wall if the intramural hematoma makes an exit in the arterial lumen [3]. While anticoagulation is used commonly in the management of acute myocardial infarction resulting from atherosclerotic coronary arterial disease it may theoretically lead to extension of the intramural hematoma in spontaneous coronary arterial dissection. This is an example highlighting why it is difficult to extrapolate the existing guidelines to the management of acute coronary syndrome in pregnancy. Similarly, the pathophysiological basis for other causes of acute coronary syndromes is also different. It is postulated that there is widespread endothelial dysfunction in pregnancy and an enhanced vascular reactivity to angiotensin II [3]. These factors lead to the occurrence of coronary arterial spasm. Some other contributing factors that play a role in the occurrence of spasm are an enhanced vascular reactivity to norepinephrine and the use of ergot derivatives to stem post-partum haemorrhage [3]. These factors are unique to pregnancy and therefore cannot be addressed by the use of existing guidelines. Finally, arterial thrombosis also occurs in pregnant females. This is brought about by a decrease in releasable tissue plasminogen activator (tPA), increased fast-acting tPA inhibitor, change in the level of coagulation factors, and reduction in functional protein $S$ levels [3]. These conditions are also unique to the pregnant female and therefore not addressed by the existing guidelines.

Pregnancy limits the use of diagnostic modalities for the diagnosis of myocardial ischemia. Nuclear imaging is contraindicated as is the use of dobutamine stress echocardiography [4]. This limits the choices to exercise stress testing with or without the use of echocardiography. While a number of investigators have established the safety of exercise in late stages of pregnancy in healthy females there is paucity in the literature on the safety of exercise in pregnant females with health problems such as hypertension and diabetes that predispose them to the development of myocardial ischemia $[5,6]$.

Important insights can be gained by studying the natural history of the various conditions that cause acute coronary syndrome in pregnant females. Spontaneous coronary arterial dissection is characterized by a high rate of spontaneous healing [7]. The response to percutaneous coronary intervention in this condition is suboptimal due to a variety of reasons. In the presence of a coronary arterial dissection it can be difficult to determine the true lumen and challenging to wire the artery without risking an extension of the dissection. These differences also highlight the inadequacies of existing literature [8].

Pregnancy and puerperium limit the choices of medications available for use in the presence of an acute coronary syndrome. For example, the existing human data on the use of thrombolytic medications in pregnancy is limited to a handful of case reports [9]. There is limited data on the use of newer antiplatelet agents such as prasugrel and ticagrelor [9]. Labetalol appears to be the only beta blocker that does not cause significant intrauterine growth restriction [9]. Drugs modulating the renin angiotensin system are universally contraindicated due to concerns about their effects on fetal renal development [9]. Only a handful of medications such as nifedipine and methyldopa can be used for the management of blood pressure [9]. 
Citation: Mallikarjuna MRE, Ganga V (2016) Unanswered Questions in Pregnancy Associated Acute Coronary Syndrome: An Argument for the Creation of Specific Guidelines for the Management of This Condition. J Health Med Inform 7: e140. doi:10.4172/2157-7420.1000e140

Page 2 of 2

The timing and mode of delivery is also controversial and does not lend it to simple generalizations [10]. While it may be safe to allow vaginal delivery in the presence of atherosclerotic coronary artery disease it is unclear if this can be allowed in the presence of coronary arterial dissection due to the fears that this might precipitate an extension.

In view of all of the above concerns we are of the opinion that the existing literature is insufficient. We argue for the creation of multicentre databases that allows the creation of large registries. We hope that a systematic analysis of such a registry will allow an evidence based approach to the management of this group of patients.

\section{References}

1. James AH, Jamison MG, Biswas MS, Brancazio LR, Swamy GK, et al. (2006) Acute myocardial infarction in pregnancy: a United States population-based study. Circulation 113: 1564-1571.

2. Elkayam U, Jalnapurkar S, Barakkat MN, Khatri N, Kealey AJ, et al. (2014) Pregnancy-Associated Acute Myocardial Infarction A Review of Contemporary Experience in 150 Cases Between 2006 and 2011. Circulation 129: 1695-1702.
3. Vijayaraghavan R, Verma S, Gupta N, Saw J (2014) Pregnancy-Related Spontaneous Coronary Artery Dissection. Circulation 130: 1915-1920.

4. European Society of Gynecology (ESG), Association for European Paediatric Cardiology (AEPC), German Society for Gender Medicine (DGesGM), Zagrosek RV, Lundqvist BC, et al. (2011) ESC Guidelines on the management of cardiovascular diseases during pregnancy. Eur Heart J 32: 3147-3197.

5. MacPhail A, Davies GA, Victory R, Wolfe LA (2000) Maximal exercise testing in late gestation: fetal responses. Obstet Gynecol 96: 565-570.

6. Szymanski LM, Satin AJ (2012) Exercise during pregnancy. fetal responses to current public health guidelines. Obstet Gynecol 119: 603-610.

7. Lettieri C, Zavalloni D, Rossini R, Morici N, Ettori F, et al. (2015) Management and Long-Term Prognosis of Spontaneous Coronary Artery Dissection. Am J Cardiol 116: 66-73.

8. Tweet MS, Eleid MF, Best PJ, Lennon RJ, Lerman A, et al. (2014) Spontaneous Coronary Artery Dissection Revascularization Versus Conservative Therapy. Circ Cardiovasc Interv 7: 777-786.

9. Briggs GG, Freeman RK (2011) Drugs in Pregnancy and Lactation: A Reference Guide to Fetal and Neonatal Risk. (10th Edn).

10. Hankins GD, Wendel GD Jr, Leveno KJ, Stoneham J (1985) Myocardial infarction during pregnancy: A review. Obstet Gynecol 65: 139-146. 